\title{
Aquaporin 1 knockdown inhibits triple-negative breast cancer cell proliferation and invasion in vitro and in vivo
}

\author{
YINAN JI ${ }^{*}$, XIAOMING LIAO* ${ }^{*}$ YI JIANG, WEI WEI and HUAWEI YANG \\ Department of Breast Surgery, Guangxi Medical University Cancer Hospital, \\ Nanning, Guangxi Zhuang Autonomous Region 530021, P.R. China
}

Received September 13, 2020; Accepted March 8, 2021

DOI: $10.3892 / 01.2021 .12698$

\begin{abstract}
Aquaporin 1 (AQP1) contributes to the progression of several cancer types, but its potential involvement in triple-negative breast cancer (TNBC) is unclear. The aim of the present study was to examine the role of AQP1 in cell proliferation and invasion in TNBC. Reverse transcription-quantitative PCR analysis and western blotting were used to detect AQP1 expression in different cell lines. A short hairpin (sh)RNA targeting AQP1 was established and transfected into MDA-MB-231 breast cancer cells. To investigate the effects of AQP1 knockdown, breast cancer cell proliferation, migration and invasion were evaluated by Cell Counting Kit- 8 and Transwell assays. Furthermore, the volume and weight of tumor xenografts in mice were measured to evaluate breast cancer growth ability. The results revealed that the levels of AQP1 were higher in the MDA-MB-231 cell line compared with those in other breast cancer cell lines (MCF-7 and SK-BR-3) and a normal mammary epithelial cell line (MCF-10A). The shRNA targeting AQP1 effectively downregulated AQP1 expression at the mRNA and protein levels, and markedly suppressed TNBC cell proliferation, migration and invasion in vitro, and tumor growth in vivo. These results suggested that AQP1 may serve as a potential therapeutic target in TNBC.
\end{abstract}

\section{Introduction}

Breast cancer is the most commonly diagnosed cancer worldwide and accounted for $\sim 25 \%$ of new cancer cases among women in 2018 (1); it is associated with a mortality

Correspondence to: Dr Huawei Yang, Department of Breast Surgery, Guangxi Medical University Cancer Hospital, 71 Hedi Road, Nanning, Guangxi Zhuang Autonomous Region 530021, P.R. China

E-mail: yanghuawei2020@outlook.com

*Contributed equally

Key words: triple-negative breast cancer, AQP1, proliferation, migration, invasion rate of $15 \%$, making it the most common cause of cancer-related mortality among women (1). The prognosis of numerous patients with breast cancer has improved considerably over the last few years with advances in hormone therapy, chemotherapy and targeted therapies (2). However, triple-negative breast cancer (TNBC), the cells of which do not express estrogen receptor, progesterone receptor or human epidermal growth factor receptor 2 , does not respond well to hormone therapy, and no effective targeted therapy is currently available (3). In addition, TNBC is aggressive and often metastasizes to distant sites (4). Therefore, the prognosis of patients with TNBC is worse compared with that of patients with other subtypes of breast cancer, and further research is needed to identify new therapeutic targets in TNBC.

Aquaporins (AQPs) are membrane proteins that transport water and small solutes across cell membranes, and play a key role in fluid homeostasis $(5,6)$. Thus far, 13 mammalian AQPs have been identified, the first of which was AQP1, which is highly expressed in the microvascular endothelium $(5,6)$. AQP1 upregulation has been reported in colon, gastric and ovarian cancer, among others, and its upregulation is associated with tumor cell replication, invasion, migration and metastasis (7-9).

AQP1 upregulation has also been reported in breast cancer, and its upregulation may be even more prominent in TNBC $(10,11)$. AQP1 may be an independent predictor of prognosis in breast cancer, and it has been proposed as a novel biomarker $(12,13)$. However, the exact function of AQP1 in breast cancer remains unclear. Therefore, the present study was undertaken to investigate the effects of AQP1 knockdown on TNBC cell proliferation, migration and invasion.

\section{Materials and methods}

Cell culture and transfection. The human breast cancer MDA-MB-231, MCF-7 and SK-BR-3 cell lines, and the human mammary epithelial MCF-10A cell line, were purchased from the Shanghai Institute of Biochemistry and Cell Biology. MDA-MB-231 and MCF-7 cells were cultured in complete DMEM supplemented with 10\% FBS (both Biological Industries), $100 \mathrm{U} / \mathrm{ml}$ penicillin and $100 \mu \mathrm{g} / \mathrm{ml}$ streptomycin. SK-BR-3 and MCF-10A cells were cultured in RPMI-1640 (Biological Industries) containing $1.5 \mathrm{mg} / \mathrm{ml} \mathrm{NaHCO}_{3}$, 
$10 \%$ FBS, $100 \mathrm{U} / \mathrm{ml}$ penicillin and $100 \mu \mathrm{g} / \mathrm{ml}$ streptomycin. All cell lines were cultured at $37^{\circ} \mathrm{C}$ in a $5 \% \mathrm{CO}_{2}$ atmosphere.

Short hairpin RNA (shRNA) targeting AQP1 mRNA (sh-AQP1; 5'-CCATTATGCTGGTGTATGT-3') (GV248-AQP1) and the corresponding negative control shRNA with a non-targeting AQP1 sequence (sh-NC; 5'-TTC TCCGAACGTGTCACGT-3') (GV248-NC) were designed and synthesized by Shanghai GeneChem Co., Ltd., and the concentrations were both adjusted to $1 \times 10^{8} \mathrm{TU} / \mathrm{ml}$. The sh-AQP1 and sh-NC $(\mathrm{MOI}=20)$ were transfected into MDA-MB-231 cells (3.0x $10^{3}$ cells per well) using polybrene (Beyotime Institute of Biotechnology) according to the manufacturer's protocol. The duration of transfection was $12 \mathrm{~h}$ at $37^{\circ} \mathrm{C}$ followed by changing the fresh medium. Transfected cells were used for subsequent experiments after $72 \mathrm{~h}$.

Reverse transcription-quantitative PCR (RT-qPCR) analysis. Total RNA was extracted from breast cancer cells (MCF-7, MDA-MB-231 and SK-BR-3) and normal mammary epithelial cells (MCF-10A) using TRIzol ${ }^{\circledR}$ reagent (Invitrogen; Thermo Fisher Scientific, Inc.), and $1 \mu \mathrm{g}$ RNA was used to generate cDNA using the ReverTra Ace qPCR RT kit (Thermo Fisher Scientific, Inc.) according to the manufacturer's instructions. qPCR was performed using the SYBR Green PCR Master mix (Takara Bio, Inc.). The following primer pairs were used: AQP1 forward, 5'-CAGCCCAAGGACAGT TCAGAG-3' and reverse, 5'-CCATCATGGCTAAGTGCA CAG-3'; and $\beta$-actin forward, 5'-TGGCACCCAGCACAA TGAA-3' and reverse, 5'-CTAAGTCATAGTCCGCCTAGA AGGA-3'. Thermocycling conditions were as follows: Initial denaturation at $95^{\circ} \mathrm{C}$ for $30 \mathrm{sec}$, followed by 40 cycles of denaturation at $95^{\circ} \mathrm{C}$ for $5 \mathrm{sec}$, annealing at $60^{\circ} \mathrm{C}$ for $30 \mathrm{sec}$ and extension at $72^{\circ} \mathrm{C}$ for $20 \mathrm{sec}$, for 40 cycles. The levels of AQP1 mRNA were quantified relative to levels of $\beta$-actin mRNA using the $2^{-\Delta \Delta \mathrm{Cq}}$ method (14).

Western blot analysis. Total protein was extracted from breast cancer cells (MCF-7, MDA-MB-231 and SK-BR-3) and normal mammary epithelial cells (MCF-10A) using RIPA lysis buffer (Beyotime Institute of Biotechnology) and measured using Pierce BCA Protein Assay kit (Beyotime Institute of Biotechnology). A total of $20 \mu \mathrm{g}$ of protein was loaded for electrophoretic separation on $10 \%$ SDS/polyacrylamide gels and transferred onto PVDF membranes (MilliporeSigma). Membranes were blocked with 5\% skimmed milk in TBS/Tween-20 (0.1\%) buffer at room temperature for $2 \mathrm{~h}$. The membranes were incubated overnight at $4^{\circ} \mathrm{C}$ with anti-AQP1 antibody (cat. no. $25287 ; 1: 100$ dilution) and anti- $\beta$-actin antibody (cat. no. 8432; 1:200 dilution) (both Santa Cruz Biotechnology, Inc.) as an internal control. Following the primary incubation, membranes were incubated with horseradish peroxidase-conjugated anti-mouse $\operatorname{IgG}$ secondary antibody (cat. no. sc-516102; 1:5,000 dilution; Santa Cruz Biotechnology, Inc.) at room temperature for $2 \mathrm{~h}$. The immunocomplex was detected using the ECL Plus kit (Amersham; Cytiva), and the band density was analyzed with Image Lab 5.0 (Bio-Rad Laboratories, Inc.).

Cell proliferation assay. Cell proliferation was measured using the Cell Counting Kit-8 (CCK-8) kit (Dojindo
Molecular Technologies, Inc.) according to the manufacturer's instructions. Untransfected MDA-MB-231 cells (blank control), and cells transfected with sh-NC or sh-AQP1 were seeded into 96-well culture plates at a density of $4 \times 10^{3}$ cells/per well and cultured at 24-h intervals for 4 days. Subsequently, $10 \mu 1$ of CCK-8 solution was added to each well and the cells were incubated for $1 \mathrm{~h}$ at $37^{\circ} \mathrm{C}$. A microplate reader (Thermo Fisher Scientific, Inc.) was used to measure the absorbance at $450 \mathrm{~nm}$.

Transwell migration and invasion assays. In the migration assay, a total of $4 \times 10^{4}$ blank control, sh-NC or sh-AQP1 cells were seeded into the upper chamber of a Transwell migration insert with $8-\mu \mathrm{m}$ pores (Corning, Inc.) containing serum-free DMEM. DMEM supplemented with 10\% FBS (600:1) was added to the lower chamber to serve as a chemoattractant. Transwell plates were incubated for $24 \mathrm{~h}$ at $37^{\circ} \mathrm{C}$, and cells that migrated to the lower chamber of the insert were fixed with 4\% paraformaldehyde, stained with Giemsa (1:10 dilution; Beijing Solarbio Science \& Technology Co., Ltd.) for $15 \mathrm{~min}$ at $37^{\circ} \mathrm{C}$ and counted under a light microscope (BX-42; Olympus Corporation; magnification, $\mathrm{x} 100$ ).

The invasion assay was performed in the same manner, except that $1 \mathrm{mg} / \mathrm{ml}$ Matrigel (BD Biosciences) was polymerized in the upper chamber prior to cell seeding.

In vivo experiments. All animal handling and experimental procedures were in compliance with and approved by the Ethics Committee of Guangxi Medical University (Nanning, China). A total of 42 female Balb/c nude mice (age, 5-6 weeks; weight, 15-16 g; n=14 mice/group) were purchased from Guangxi Medical University Animal Experiment Center and maintained under specific pathogen-free conditions at $22-25^{\circ} \mathrm{C}$ and a $12-\mathrm{h} \mathrm{light/dark} \mathrm{cycle,} \mathrm{with} \mathrm{free} \mathrm{access} \mathrm{to}$ water and sterile food. Previously transfected blank control, sh-NC or sh-AQP1 cells were suspended in PBS, the density was adjusted to $1 \times 10^{7}$ cells $/ \mathrm{ml}$ and then $100 \mu \mathrm{l}$ of the suspension was injected subcutaneously under the breast fat pad of the nude mice. The tumor volume was measured every 5 days using a vernier caliper and calculated according to the following formula: (Length $\mathrm{x}$ width $\left.{ }^{2}\right) / 2$. The maximum tumor volume observed in the present study was $1,102.6 \mathrm{~mm}^{3}$. After 5 weeks, the mice were anesthetized by intraperitoneal injection with $60 \mu 110 \%$ chloral hydrate $(300 \mathrm{mg} / \mathrm{kg}$; Beyotime Institute of Biotechnology) and sacrificed by cervical dislocation. No mice exhibited signs of peritonitis, pain or discomfort. Death was verified by confirming respiratory and cardiac arrest.

Statistical analysis. All data are reported as the mean \pm SD. Multiple group comparisons were performed using a one-way ANOVA followed by Bonferroni's post hoc test. $\mathrm{P}<0.05$ was considered to indicate a statistically significant difference. All statistical analyses were performed using SPSS 19.0 (IBM Corp.).

\section{Results}

AQP1 expression in breast cancer cell lines. In the present study, the expression levels of AQP1 in the human breast cancer MDA-MB-231, MCF-7 and SK-BR-3 cell lines, as 
A

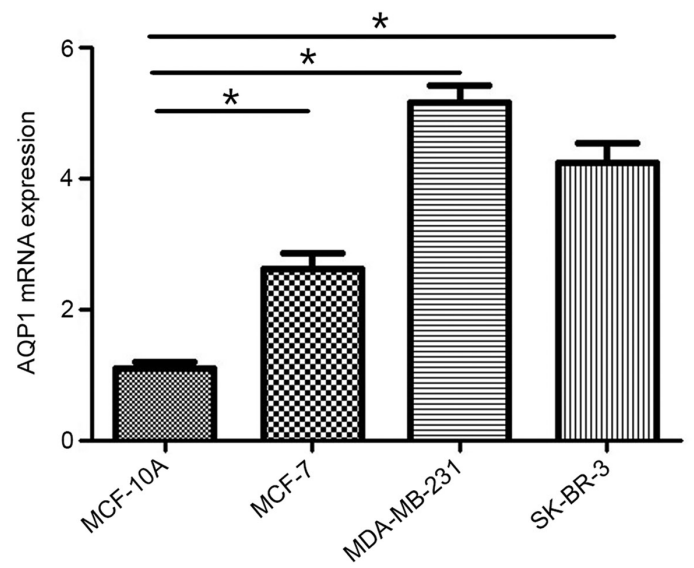

B

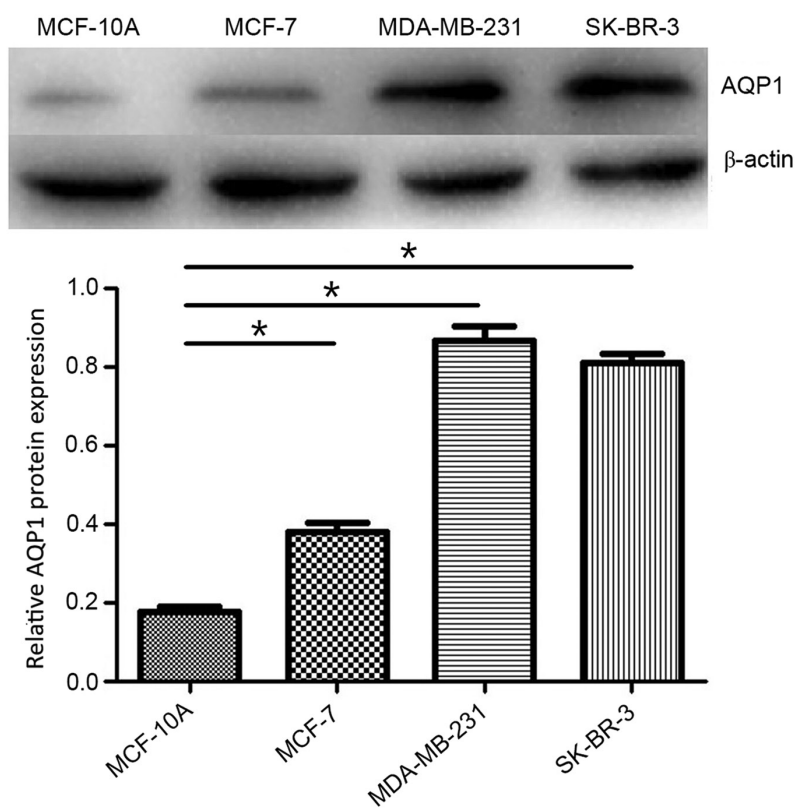

Figure 1. AQP1 expression in breast cancer cell lines. (A) The mRNA expression of AQP1 in three human breast cancer cell lines, MCF-7, MDA-MB-231 and SK-BR-3, as well as the normal human mammary epithelial cell line, MCF-10A, was evaluated using reverse transcription-quantitative PCR analysis. (B) The protein expression of AQP1 in MCF-7, MDA-MB-231 and SK-BR-3 cells, as well as in MCF-10A cells, was evaluated using western blotting. Results were obtained from three independent experiments. ${ }^{*} \mathrm{P}<0.05$. AQP1, aquaporin 1.

well as in the normal human mammary epithelial MCF-10A cell line, were determined by western blotting and RT-qPCR analyses. As shown in Fig. 1A, AQP1 mRNA expression was significantly higher in MDA-MB-231, MCF-7 and SK-BR-3 cells compared with that in MCF-10A cells. Similarly, western blotting demonstrated higher protein expression levels of AQP1 in MDA-MB-231, MCF-7 and SK-BR-3 cells compared with those in MCF-10A cells (Fig. 1B). The levels of AQP1 were higher in the TNBC MDA-MB-231 cell line compared with those in the other breast cancer cell lines. Thus, the MDA-MB-231 cell line was used to investigate the effect of AQP1 on breast cancer cell proliferation, invasion and migration.

AQP1 knockdown inhibits TNBC cell proliferation, invasion and migration in vitro. To assess how AQP1 affects cell

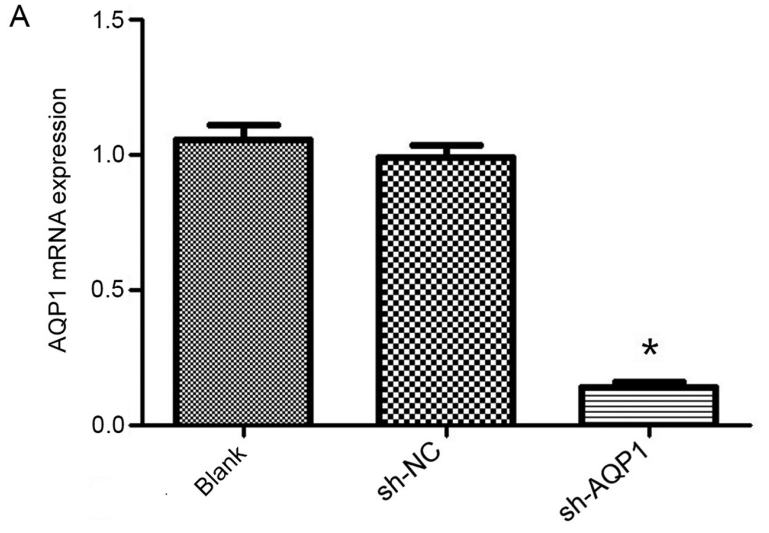

B
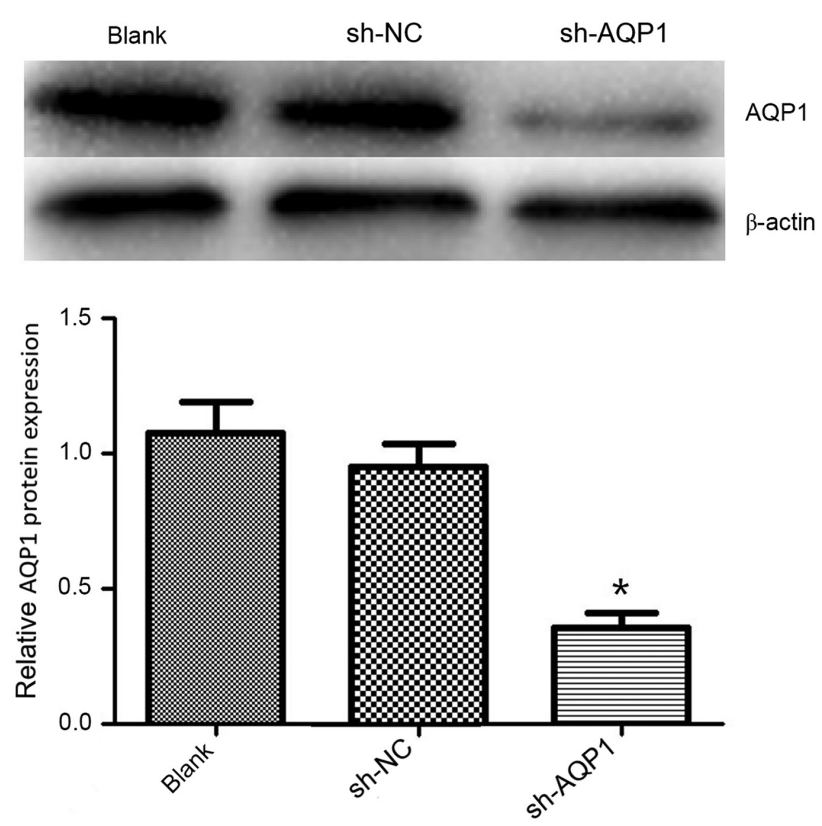

Figure 2. AQP1 expression in MDA-MB-231 cells following transfection. (A) Expression of AQP1 mRNA in untransfected MDA-MB-231 cells (blank control), and cells transfected with sh-NC or sh-AQP1. (B) Expression of AQP1 protein in blank control, sh-NC or sh-AQP1 MDA-MB-231 cells. Results were obtained from three independent experiments. ${ }^{*} \mathrm{P}<0.05$ vs. NC. AQP1, aquaporin 1; NC, negative control; shRNA, short hairpin RNA.

proliferation in vitro, shRNA was used to knock down AQP1 expression in MDA-MB-231 cells. The expression of AQP1 at the mRNA (Fig. 2A) and protein (Fig. 2B) levels was significantly decreased in MDA-MB-231 cells post-transfection. The CCK- 8 proliferation assay was subsequently applied to assess the effect of AQP1 knockdown on the proliferation of breast cancer cells, and it was found that AQP1 knockdown led to a significantly lower level of proliferation compared with that in blank control cells and cells treated with sh-NC (Fig. 3A).

Transwell migration and invasion assays were performed to investigate the effects of AQP1 knockdown on the migration and invasion of breast cancer cells. Compared with that in the blank control and sh-NC groups, a significant decrease in the number of migrating (Fig. 3B) and invading (Fig. 3C) MDA-MB-231 cells was apparent following transfection with sh-AQP1. 
A

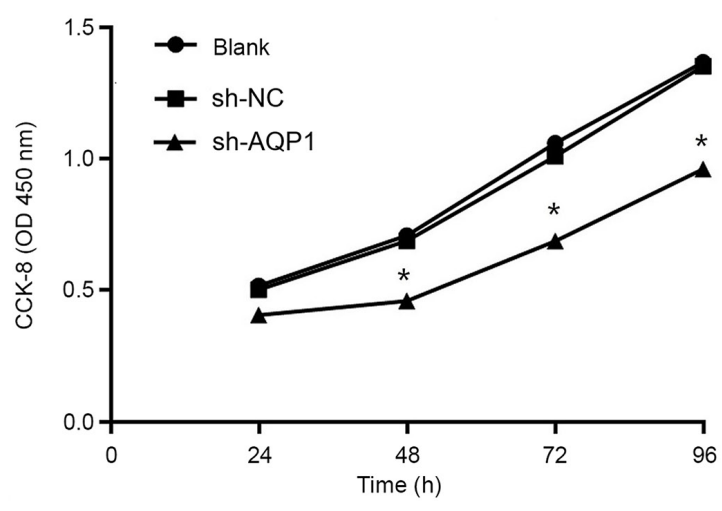

B

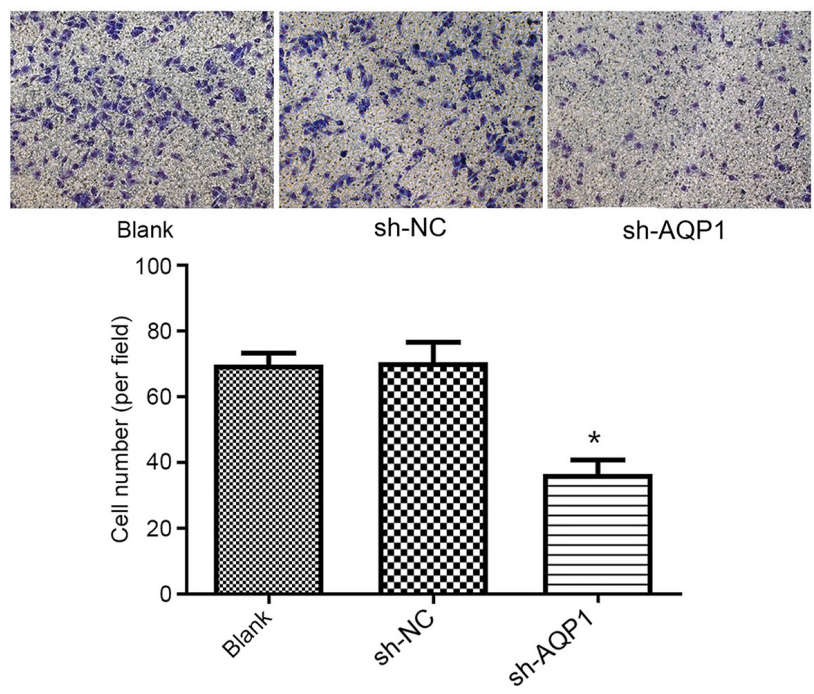

C
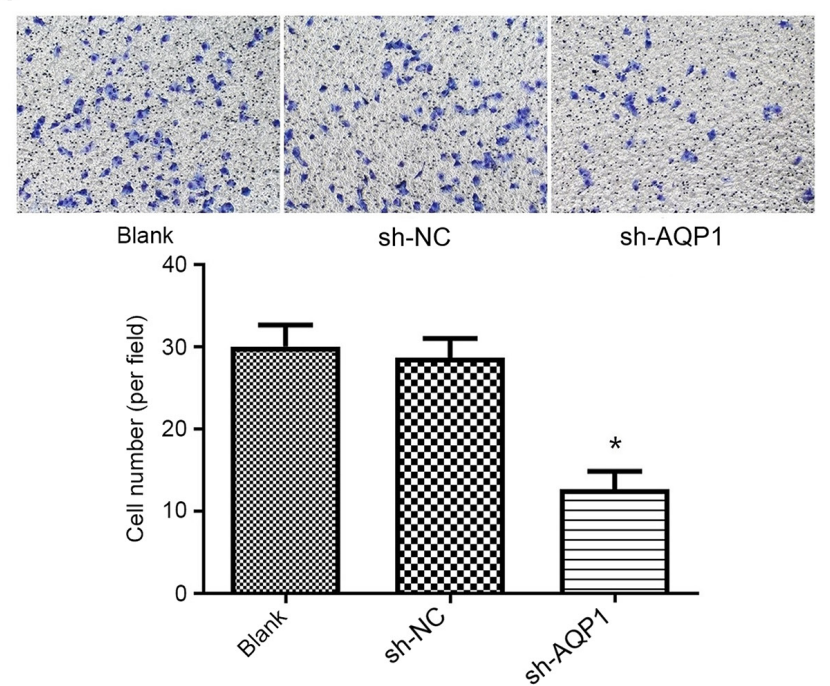

Figure 3. Knockdown of AQP1 inhibits the proliferation, migration and invasion of breast cancer cells. MDA-MB-231 cells were untransfected (blank control), or transfected with sh-NC or sh-AQP1 for $24 \mathrm{~h}$. (A) Cell proliferation was evaluated using the CCK- 8 proliferation assay. (B) Cell migration was detected using the Transwell migration assay. (C) Cell invasion was detected using the Transwell invasion assay. Results were obtained from three independent experiments. ${ }^{*} \mathrm{P}<0.05$ vs. NC. AQP1, aquaporin 1 ; NC, negative control; shRNA, short hairpin RNA; OD, optical density; CCK-8, Cell Counting Kit- 8 .

AQP1 knockdown suppresses TNBC xenograft growth in vivo. In order to confirm the potential antitumor effects of AQP1 downregulation in vivo, female $\mathrm{BALB} / \mathrm{c}$ nude mice were

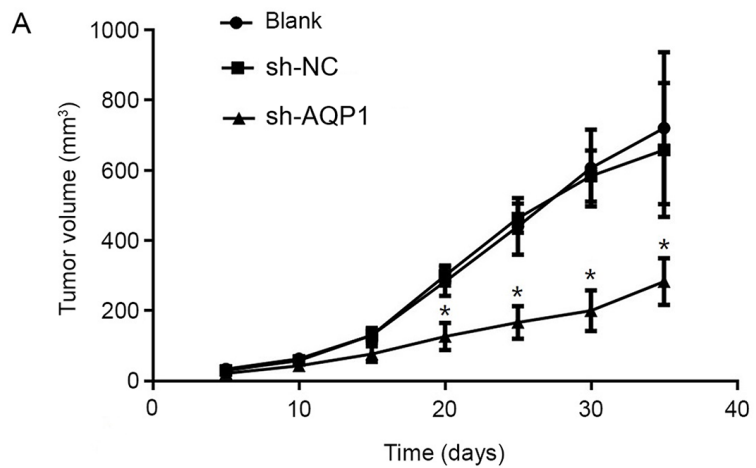

B
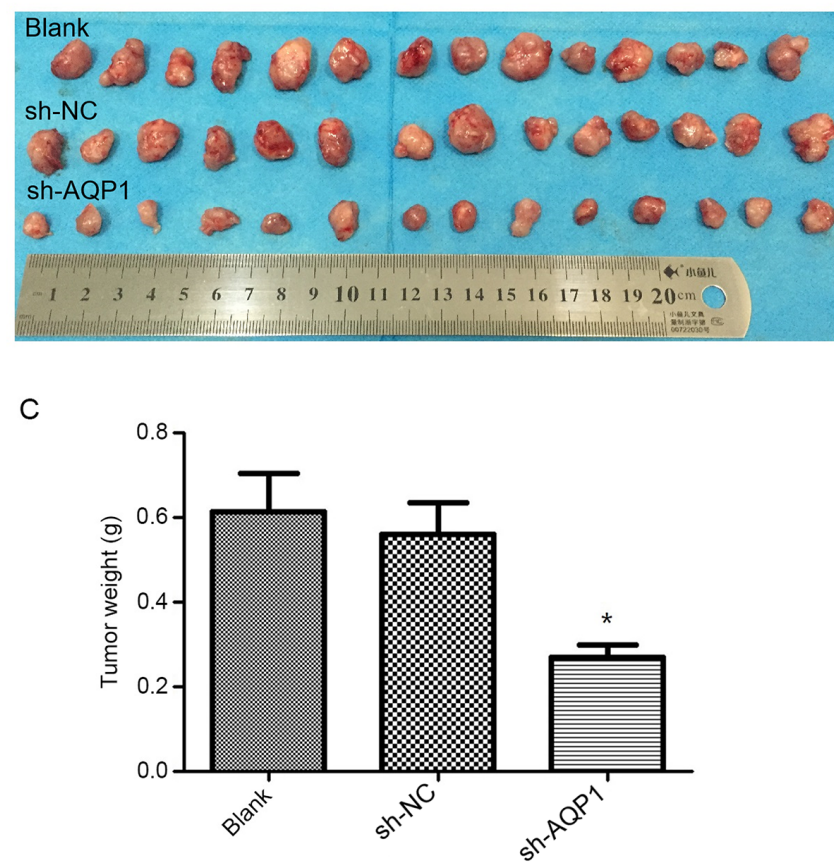

Figure 4. Knockdown of AQP1 attenuates xenograft tumor growth in vivo. Blank control, sh-NC or AQP1-shRNA cells were suspended in PBS, and $1 \times 10^{7}$ cells $/ \mathrm{ml}$ in $100 \mu \mathrm{l}$ was injected subcutaneously under the breast fat pad of female, 6-week-old BALB/c nude mice. (A) Tumor size was monitored weekly. (B and C) After 4 weeks, the mice were sacrificed, and tumors were collected and weighed. "P<0.05 vs. NC. AQP1, aquaporin 1; NC, negative control; shRNA, short hairpin RNA.

subcutaneously injected with MDA-MB-231 cells that were stably transfected with sh-AQP1. When comparing tumor sizes, the knockdown of AQP1 was found to significantly decrease tumor growth compared with the blank control and sh-NC groups (Fig. 4A and B). Furthermore, AQP1 knockdown significantly decreased the weight of the xenografted tumors (Fig. 4C).

\section{Discussion}

The findings of the present study confirmed that AQP1 was overexpressed in breast cancer cells compared with that in normal mammary epithelial cells, which was in agreement with previous findings (15). It was also confirmed that AQP1 was expressed at higher levels in the TNBC MDA-MB-231 cell line compared with that in other types of breast cancer cells, which was consistent with a previous study reporting a strong association between AQP1 expression and high tumor 
grade and hormone receptor negativity (11). A previous study showed that overexpression of AQP1 promoted the proliferation and invasion of breast cancer cells (12). The result suggested that AQP1 could be a potential prognostic biomarker for breast cancer. However, it is not clear whether AQP1 can be used as a potential therapeutic target in breast cancer, particularly TNBC. Thus, to explore the antitumor potential of downregulating AQP1, the present study investigated the effects of AQP1 knockdown on TNBC growth in vitro and in vivo.

It was also demonstrated that AQP1 knockdown inhibited the proliferation of TNBC cells, which was consistent with the results of previous studies in which AQP1 knockdown markedly suppressed the viability and promoted the apoptosis of ovarian cancer cells (16), and suppressed the proliferation of lung adenocarcinoma, osteosarcoma and glioma cells (17-19). However, AQP1 downregulation did not alter the proliferation of B16F10 melanoma cells, although it did alter their water permeability (20). These results suggest that AQP1 plays different roles in different types of cancer.

Breast cancer invasiveness decreases patient survival and doubles the mortality rate (21). The present study demonstrated that AQP1 knockdown suppressed the migration and invasion of TNBC cells in vitro, as previously reported for ovarian cancer, lung adenocarcinoma, osteosarcoma and glioma cells in culture (16-19), but not for melanoma cells (20). AQP1 overexpression may help drive tumor cell migration by importing water to fill cellular protrusions, creating more space for actin polymerization at the leading edge of migration (22-24). AQP1 may also promote tumor invasion by stimulating angiogenesis (25-27).

Regardless of the underlying mechanism, AQP1 may serve as a potential therapeutic target in TNBC by inhibiting the proliferation, migration and invasion of tumor cells.

\section{Acknowledgements}

Not applicable.

\section{Funding}

The present study was supported by the National Natural Science Foundation of China (grant no. 81860464) and the Natural Science Foundation of Guangxi Province (grant no. 2019GXNSFAA185038)

\section{Availability of data and materials}

The datasets used and/or analyzed during the present study are available from the corresponding author on reasonable request.

\section{Authors' contributions}

HY conceived and designed the study. YJ and XL performed the experiments. YNJ and WW analyzed the data. YJ wrote the manuscript. YNJ and HY confirm the authenticity of all the raw data. All the authors have read and approved the final manuscript, and agree to be accountable for all aspects of the research.

\section{Ethics approval and consent to participate}

All animal handling and experimental procedures were in compliance with and approved by the Ethics Committee of the Guangxi Medical University (Nanning, China).

\section{Patient consent for publication}

Not applicable.

\section{Competing interests}

The authors declare that they have no competing interests.

\section{References}

1. Bray F, Ferlay J, Soerjomataram I, Siegel RL, Torre LA and Jemal A: Global cancer statistics 2018: GLOBOCAN estimates of incidence and mortality worldwide for 36 cancers in 185 countries. CA Cancer J Clin 68: 394-424, 2018.

2. DeSantis CE, Ma J, Goding Sauer A, Newman LA and Jemal A: Breast cancer statistics, 2017, racial disparity in mortality by state. CA Cancer J Clin 67: 439-448, 2017.

3. Scott LC, Mobley LR, Kuo TM and Il'yasova D: Update on triple-negative breast cancer disparities for the United States: A population-based study from the United States cancer statistics database, 2010 through 2014. Cancer 125: 3412-3417, 2019.

4. Hwang KT, Kim J, Jung J, Chang JH, Chai YJ, Oh SW, Oh S, Kim YA, Park SB and Hwang KR: Impact of breast cancer subtypes on prognosis of women with operable invasive breast cancer: A population-based study using SEER database. Clin Cancer Res 25: 1970-1979, 2019.

5. Verkman AS and Mitra AK: Structure and function of aquaporin water channels. Am J Physiol Renal Physiol 278: F13-F28, 2000.

6. Agre P, King LS, Yasui M, Guggino WB, Ottersen OP, Fujiyoshi Y, Engel $A$ and Nielsen S: Aquaporin water channels-from atomic structure to clinical medicine. J Physiol 542: 3-16, 2002.

7. Kang BW, Kim JG, Lee SJ, Chae YS, Jeong JY, Yoon GS, Park SY, Kim HJ, Park JS, Choi GS and Jeong JY: Expression of aquaporin-1, aquaporin-3, and aquaporin-5 correlates with nodal metastasis in colon cancer. Oncology 88: 369-376, 2015.

8. Thapa S, Chetry M, Huang K, Peng Y, Wang J, Wang J, Zhou Y, Shen Y, Xue Y and Ji K: Significance of aquaporins' expression in the prognosis of gastric cancer. Biosci Rep 38: BSR20171687, 2018.

9. Chetry M, Li S, Liu H, Hu X and Zhu X: Prognostic values of aquaporins mRNA expression in human ovarian cancer. Biosci Rep 38: BSR20180108, 2018.

10. Otterbach F, Callies R, Kimmig R, Schmid KW and Bánkfalvi A: Aquaporin 1 expression in invasive breast carcinomas. Pathologe 29 (Suppl 2): S357-S362, 2008 (In German).

11. Otterbach F, Callies R, Adamzik M, Kimmig R, Siffert W, Schmid KW and Bankfalvi A: Aquaporin 1 (AQP1) expression is a novel characteristic feature of a particularly aggressive subgroup of basal-like breast carcinomas. Breast Cancer Res Treat 120: 67-76, 2010

12. Qin F, Zhang H, Shao Y, Liu X, Yang L, Huang Y, Fu L, Gu F and Ma Y: Expression of aquaporin1, a water channel protein, in cytoplasm is negatively correlated with prognosis of breast cancer patients. Oncotarget 7: 8143-8154, 2016.

13. Zhu L, Ma N, Wang B, Wang L, Zhou C, Yan Y, He J and Ren Y: Significant prognostic values of aquaporin mRNA expression in breast cancer. Cancer Manag Res 11: 1503-1515, 2019.

14. Livak KJ and Schmittgen TD: Analysis of relative gene expression data using real-time quantitative PCR and the 2(-Delta Delta C(T)) method. Methods 25: 402-408, 2001.

15. Shi Z, Zhang T, Luo L, Zhao H, Cheng J, Xiang J and Zhao C: Aquaporins in human breast cancer: Identification and involvement in carcinogenesis of breast cancer. J Surg Oncol 106: 267-272, 2012.

16. Wang Y, Fan Y, Zheng C and Zhang X: Knockdown of AQP1 inhibits growth and invasion of human ovarian cancer cells. Mol Med Rep 16: 5499-5504, 2017.

17. Wei $\mathrm{X}$ and Dong J: Aquaporin 1 promotes the proliferation and migration of lung cancer cell in vitro. Oncol Rep 34: 1440-1448, 2015. 
18. Guan Y, Chen J, Zhan Y and Lu H: Effects of dexamethasone on C6 cell proliferation, migration and invasion through the upregulation of AQP1. Oncol Lett 15: 7595-7602, 2018.

19. Wu Z, Li S, Liu J, Shi Y, Wang J, Chen D, Luo L, Qian Y, Huang X and Wang H: RNAi-mediated silencing of AQP1 expression inhibited the proliferation, invasion and tumorigenesis of osteosarcoma cells. Cancer Biol Ther 16: 1332-1340, 2015.

20. $\mathrm{Hu} \mathrm{J}$ and Verkman AS: Increased migration and metastatic potential of tumor cells expressing aquaporin water channels. FASEB J 20: 1892-1894, 2006.

21. Hussain SA, Ganesan R, Reynolds G, Gross L, Stevens A,Pastorek J, Murray PG, Perunovic B, Anwar MS, Billingham L, et al: Hypoxia-regulated carbonic anhydrase IX expression is associated with poor survival in patients with invasive breast cancer. $\mathrm{Br}$ J Cancer 96: 104-109, 2007.

22. Verkman AS: Aquaporin water channels and endothelial cell function. J Anat 200: 617-627, 2002.

23. Verkman AS: Knock-out models reveal new aquaporin functions. Handb Exp Pharmacol: 359-381, 2009.

24. Verkman AS, Hara-Chikuma $M$ and Papadopoulos MC: Aquaporins-new players in cancer biology. J Mol Med (Berl) 86 523-529, 2008.
25. Esteva-Font C, Jin BJ and Verkman AS: Aquaporin-1 gene deletion reduces breast tumor growth and lung metastasis in tumor-producing MMTV-PyVT mice. FASEB J 28: 1446-1453, 2014.

26. Nicchia GP, Stigliano C, Sparaneo A, Rossi A, Frigeri A and Svelto M: Inhibition of aquaporin-1 dependent angiogenesis impairs tumour growth in a mouse model of melanoma. J Mol Med (Berl) 91: 613-623, 2013.

27. El Hindy N, Bankfalvi A, Herring A, Adamzik M, Lambertz N, Zhu Y, Siffert W, Sure U and Sandalcioglu IE: Correlation of aquaporin-1 water channel protein expression with tumor angiogenesis in human astrocytoma. Anticancer Res 33: 609-613, 2013. International (CC BY-NC-ND 4.0) License. 